\title{
Effect of Influenza Vaccination on Risk of Stroke: A Systematic Review and Meta-Analysis
}

\author{
Kyu Rae Lee ${ }^{a}$ Jeong Hun Bae ${ }^{b}$ In Cheol Hwang ${ }^{a}$ Kyoung Kon Kim ${ }^{a}$ \\ Heuy Sun Suh ${ }^{\mathrm{a}}$ Ki Dong Ko ${ }^{\mathrm{a}}$ \\ ${ }^{a}$ Department Family Medicine, Gachon University Gil Medical Center, Incheon, and bepartment of Ophthalmology, \\ Kangbuk Samsung Hospital, Sungkyunkwan University School of Medicine, Seoul, Republic of Korea
}

\section{Keywords}

Influenza vaccination · Influenza $\cdot$ Stroke

\begin{abstract}
Background: Despite the presence of a strong association between influenza infection and stroke, whether influenza vaccination reduces the risk of stroke is yet a matter of controversy. We conducted a meta-analysis to determine whether influenza vaccination protects against stroke. Methods: We searched PubMed, EMBASE, and the Cochrane Library from database inception date to November 18, 2016, without language restrictions, to identify studies investigating the effect of influenza vaccination on subsequent risk of stroke. We conducted a meta-analysis to quantify the risk of stroke in overall and subgroup analyses and calculated a pooled OR for developing stroke with a $95 \% \mathrm{Cl}$. Publication bias was assessed by Begg's rank correlation test. Results: Eleven studies fulfilled our inclusion criteria. In a randomeffects model, vaccinated individuals had a decreased risk of stroke compared with unvaccinated individuals (OR 0.82; $95 \% \mathrm{Cl} 0.75-0.91 ; p<0.001)$. The relationship between influenza vaccination and stroke risk remained robust in subgroup analyses. The significant effect of influenza vaccination was associated with ascertainment of vaccination status and stage of prevention. Conclusion: Vaccination against in-
\end{abstract}

\section{KARGER}

๑) 2017 S. Karger AG, Basel

E-Mail karger@karger.com

www.karger.com/ned fluenza is associated with a lower risk of stroke. Well-designed prospective studies are needed to provide stronger evidence of the protective effect of influenza vaccination against stroke.

(c) 2017 S. Karger AG, Basel

\section{Introduction}

Stroke is a leading cause of death worldwide and can also result in long-term disability; about 5 million stroke survivors are alive today [1]. Over the last 30 years, the age-adjusted incidence of stroke has declined, but the aging of the population suggests that absolute numbers of stroke will increase over the next decades [2]. To prevent stroke, it is important to identify and modify risk factors. Besides conventional risk factors for stroke (i.e., increasing age, hypertension, smoking, diabetes, and elevated cholesterol), current evidence has raised the possibility that other risk factors, such as infection, also play a role [3].

Cardiovascular disease (CVD) including stroke is more common in the winter and during influenza epi-

K.R.L. and J.H.B. contributed equally to this paper as first author.
In Cheol Hwang, MD, MPH, PhD

Gachon University Gil Medical Center 1198 Guwol-dong, Namdong-gu

Incheon 405-760 (Republic of Korea)

E-Mail spfe0211@gmail.com 
demics, which could partially be explained by seasonal changes in metabolic risk factors or cold-induced vascular stress $[4,5]$. However, a large body of evidence suggests that influenza itself could be a trigger of major CVD, further adding to the morbidity and mortality associated with this infection. A recent meta-analysis [6] shows that influenza infection is significantly associated with acute myocardial infarction, and a similar association with stroke was reported in several previous studies $[7,8]$. This poses the question of whether immunizing against influenza could reduce the risk of major CVD.

Of late, there is growing interest in the use of influenza vaccines for preventing CVD, primarily coronary artery disease In mouse models, influenza vaccination protects against acute myocardial infarction, with reduced atherosclerotic plaque size, increased plaque stability, and decreased pro-inflammatory markers [9]. An increasing body of evidence suggests that influenza vaccines protect against heart disease in terms of both primary prevention and secondary prevention $[6,10]$. However, associations with the risk of stroke have been investigated in limited epidemiological studies and their results have been inconsistent. Whereas some studies showed that influenza vaccination, either alone $[8,11-$ 13] or combined with pneumococcal vaccine [14], was associated with reduced risk of stroke, other studies did not [15].

Many countries recommend annual influenza vaccination for elderly people and/or those with CVD, which are risk groups for serious influenza infection. Also, influenza vaccination is estimated to be a cost-effective method of influenza prevention among older adults without direct consideration of CVD protection [16]. If influenza vaccination has a protective effect against stroke, this could increase the rate of vaccinations, which are currently underused [17]. Thus, we conducted a meta-analysis of studies investigating the protective effect of influenza vaccination against stroke.

\section{Methods}

\section{Search Strategy}

We searched PubMed, EMBASE, and the Cochrane Library from the databases' inception to November 18, 2016, without language restrictions. Search terms were related to stroke (stroke or cerebrovascular disease or cerebrovascular accident or transient ischemic accident) and influenza vaccination (influenza vaccine or influenza vaccination or flu vaccine or flu vaccination). We also reviewed the reference lists of relevant articles to identify additional papers not captured in the database searches. Our review followed the guidelines for meta-analysis of observational studies in epidemiology [18]. Ethical approval was not required because the data included in this study were extracted from published literature.

\section{Study Selection and Inclusion Criteria}

We included original studies of the association between influenza vaccination and stroke risk in humans. Eligibility criteria were the reporting of the risk of stroke (i.e., not only stroke-related mortality) and measures of association (e.g., hazard ratio, incidence ratio, OR) and precision (e.g., CI) irrespective of study design. We collected the fully adjusted measures of association. In the case of multiple publications based on the same study sample, the most relevant publication was included in the analysis. As an individual's risk of CVD and likelihood of receiving a vaccine is known to increase over an observation period [19], we considered stroke data only from the year that the vaccination was received $[13,20]$.

\section{Data Extraction}

All potentially relevant articles were independently evaluated by 2 investigators, and disagreements were resolved by consensus or consultation with a third author. The following data were extracted from each study: first author, year of publication, country, study population and setting, study design, stroke subtype, method of ascertainment of vaccination status, stage of prevention, measures of association with corresponding 95\% CIs, and core-adjusted variables. Some estimates had to be recalculated (e.g., pooling the estimates of 2 cohorts [12] and those for various time periods after vaccination [8]). We used the Newcastle-Ottawa Scale (NOS) to assess the methodological quality of studies (online suppl. Table 1, see www.karger. com/doi/10.1159/000478017). Low quality was defined as an NOS score $\leq 7$ and high quality as an NOS score $\geq 8$ (maximum score, 9). Further, we stratified articles by their risk of selection bias based on core-adjusted variables into low, moderate, or high risk.

\section{Statistical Analysis}

We investigated the effect of influenza vaccination on subsequent risk of stroke. Subgroup analyses were performed depending on the methodological quality (low or high), the risk of selection bias (low risk or moderate to high risk), method of ascertainment of vaccination status (computerized database or recall), stage of prevention (first stroke, recurrent stroke, or any stroke), and stroke subtype (all types or ischemic stroke). We calculated a pooled OR with a $95 \%$ CI from those reported in the individual studies. We assumed that the other relative measures were close to the OR because the stroke incidence was sufficiently low for the rare disease assumption to apply [21]. To calculate the variance of $\log$ OR from each study, we converted the $95 \%$ CI to its natural logarithm by taking the width of the CI and dividing it by 3.92. To test for heterogeneity, we used Higgins $I^{2}$, which indicates the percentage of variation across studies (0-100\%). When substantial heterogeneity $\left(I^{2}>50 \%\right)$ was observed, we used the DerSimonian and Laird random-effects model, which is the generally preferred approach. Publication bias was assessed using the Begg's rank correlation test. All statistical analyses were performed with Stata version 12.1 (StataCorp, College Station, TX, USA). 
Fig. 1. Flow diagram for identifying relevant articles.

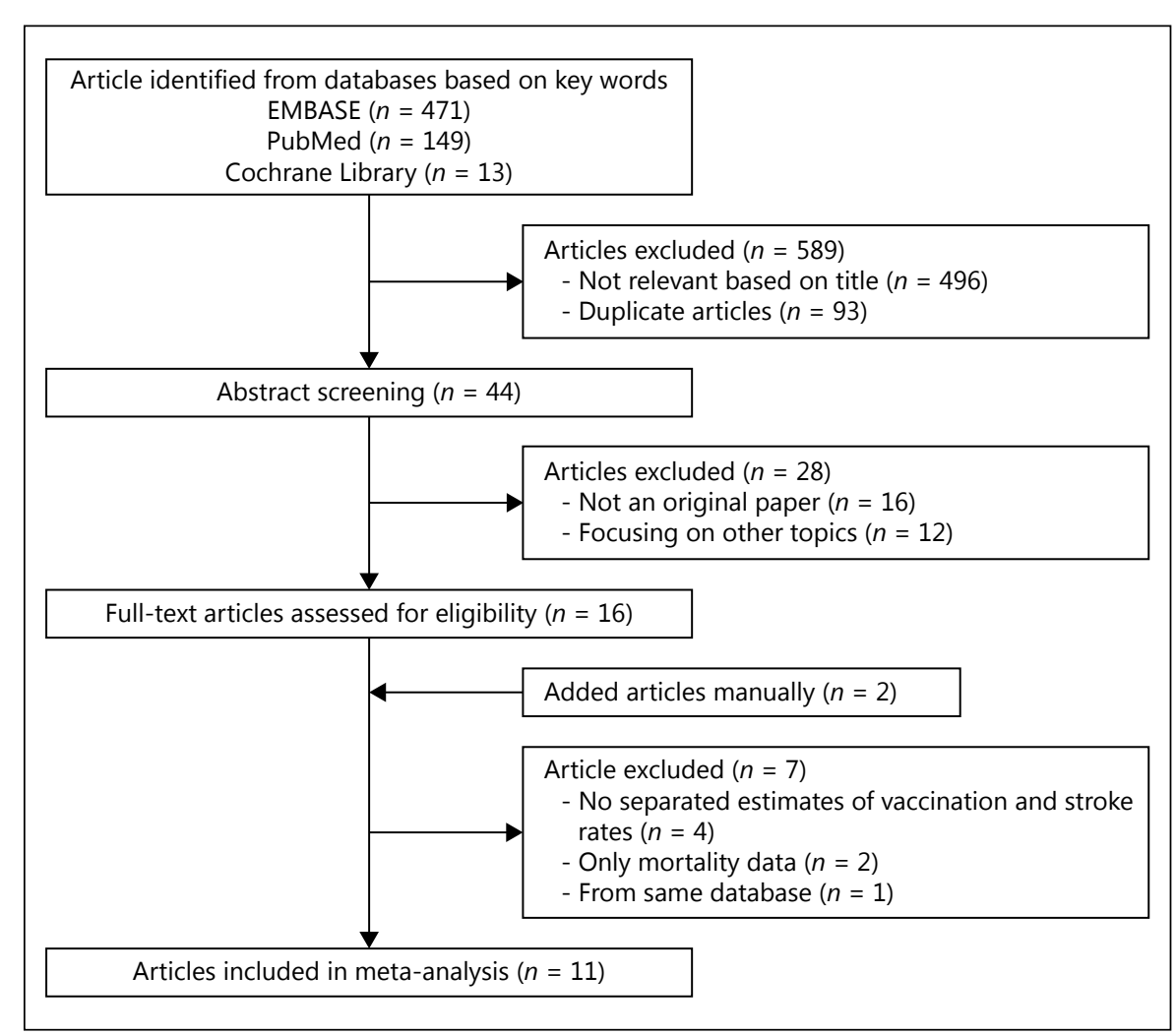

\section{Results}

Figure 1 shows a flow diagram for the identification of relevant studies. Of the 633 articles retrieved, 44 underwent abstract review, and 16 of these met our eligibility criteria. After reviewing the full text of these 16 articles, we excluded 7 articles for the following reasons: no separate estimate of influenza vaccination or on stroke $(n=4)$, only mortality data presented $(n=2)$, or the data were from a sample described by another study included in our analysis $(n=1)$. After adding 2 studies identified through manual searches, a total of 11 studies were included in the final analysis. Of 3 studies sampled from the United Kingdom General Practice Research Database, 2 studies included overlapping groups of participants [22, 23]; we used data from each study in overall and subgroup analyses as appropriate.

Characteristics of the included studies are summarized in Table 1. Most were conducted in western countries $(n=9)$. Two studies $[14,24]$ were prospective intervention designs. Half of the studies were limited to elderly people $(n=5)$, and half of the studies reliably ascertained vaccination status $(n=6)$. Information on stroke subtype (i.e., all strokes, ischemic stroke, and hemorrhagic stroke) varied across studies. The risk of selection bias was as- sessed based on core-adjusted variables, and 7 studies $[8$, $11,13,14,23-25]$ were deemed to have a low risk of bias.

Overall, vaccinated individuals had a decreased risk of stroke (OR 0.82; 95\% CI 0.75-0.91; $p<0.001$; Fig. 2). No single study had an unduly large influence on the results. The association between influenza vaccination and stroke risk remained robust in most subgroup analyses; some did not reach significance (Table 2). Funnel plot asymmetry among studies was not observed among the studies $(p=0.299$; Fig. 3).

\section{Discussion}

Given that influenza infection is a trigger of stroke, a logical and appealing inference is that influenza vaccination could prevent stroke. Influenza vaccination has been approved as a simple protective strategy for reducing influenza-related complications [26]. In this meta-analysis, we found that vaccinated people have a decreased risk of developing stroke, which has considerable clinical and health policy implications given the profound underuse of vaccinations. This finding also suggests that influenza itself and/or its associated morbidity could be an underrecognized residual CVD risk factor. 


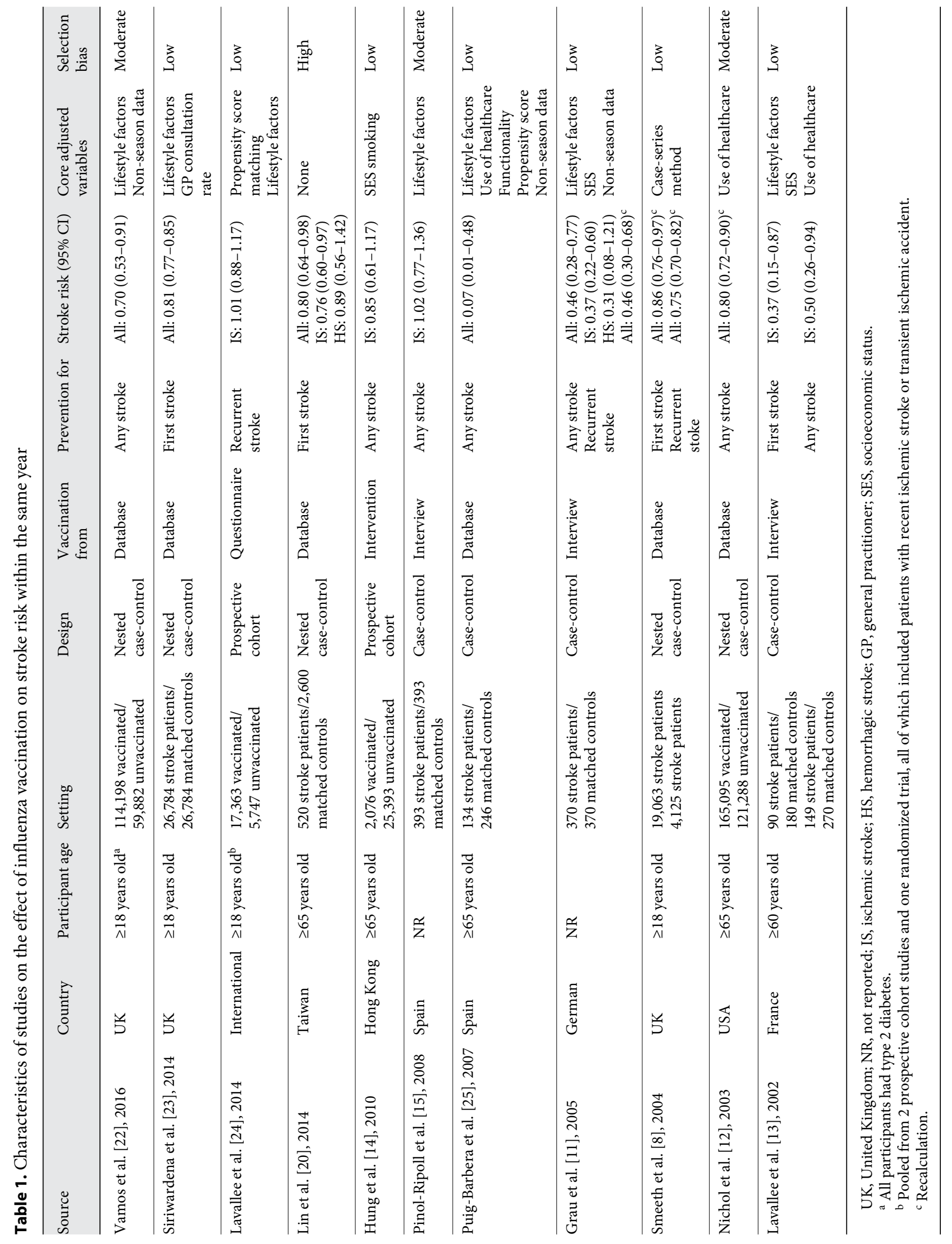


Table 2. Stroke risk among individuals who received influenza vaccination

\begin{tabular}{|c|c|c|c|c|}
\hline & Number of studies & Summary OR (95\% CI) & Heterogeneity, $I^{2}$, \% & Model \\
\hline Overall $[8,11-15,20,23-25]$ & 10 & $0.82(0.75-0.91)$ & 63.5 & Random-effect \\
\hline \multicolumn{5}{|l|}{ Study quality ${ }^{\mathrm{a}}$} \\
\hline High $[8,14,23]$ & 3 & $0.81(0.77-0.85)$ & 0 & Fixed-effect \\
\hline \multicolumn{5}{|l|}{ Risk of selection bias } \\
\hline Low $[8,11,13,14,23-25]$ & 7 & $0.79(0.68-0.92)$ & 72.9 & Random-effect \\
\hline \multicolumn{5}{|l|}{ Vaccination status } \\
\hline Confirmed $[8,12,14,20,23,25]$ & 6 & $0.81(0.77-0.84)$ & 20.6 & Fixed-effect \\
\hline By recall $[11,13,15,24]$ & 4 & $0.77(0.55-1.09)$ & 76.3 & Random-effect \\
\hline \multicolumn{5}{|l|}{ Prevention for } \\
\hline First stroke $[8,13,20,23]$ & 4 & $0.81(0.78-0.85)$ & 23.8 & Fixed-effects \\
\hline Any stroke $[11-15,22,25]$ & 7 & $0.73(0.59-0.90)$ & 63.5 & Random-effects \\
\hline
\end{tabular}

Fig. 2. Forest plot showing the effect of influenza vaccination on risk of stroke. OR, odds ratio; $\mathrm{CI}$, confidence interval.

Fig. 3. Begg's funnel plot with $95 \%$ confidence limits for incident stroke among the included studies. OR, odds ratio.

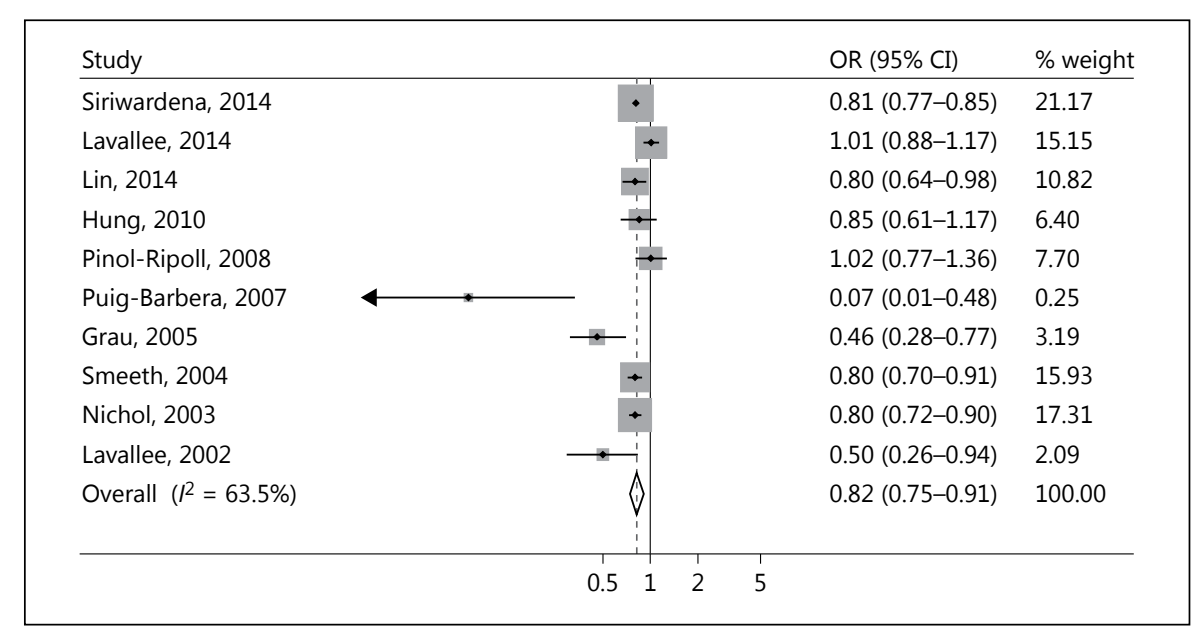

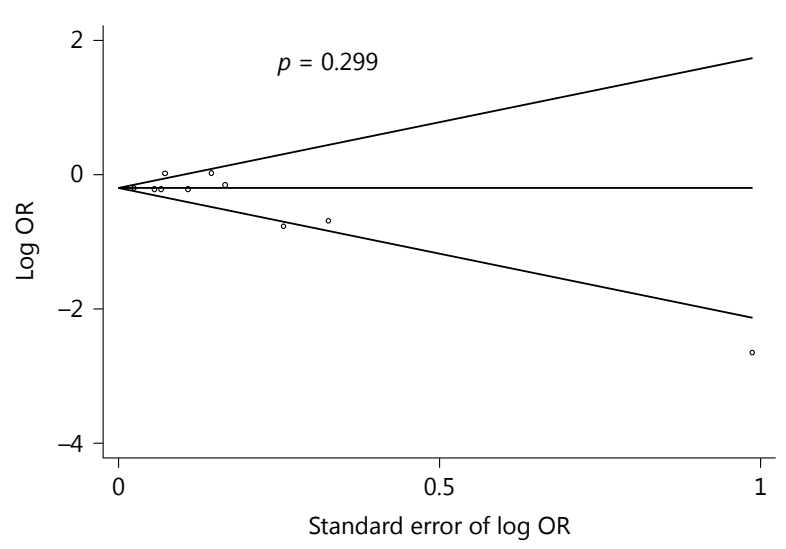

Neuroepidemiology 2017;48:103-110 DOI: $10.1159 / 000478017$ 
The question of whether influenza vaccination has a preventive effect against stroke can be definitively answered only with a trial design. Although a small randomized controlled trial sought this question, stroke events were too rare to analyze [27]. Instead, most previous studies have employed an observational design, which is susceptible to many potential biases. A crucial but inevitable flaw in observational studies is the presence of systematic differences between vaccine recipients and nonrecipients [28]. Attitudes toward health issues, especially for primary prevention such as vaccination, are tightly associated with health consciousness and socioeconomic factors, and healthier people at lower risk of stroke are more likely to be vaccinated [29]. To minimize this "healthy vaccine" bias, researchers have adjusted for other preventive (e.g., a blood cholesterol assay) and lifestyle factors in their analyses. Indeed, our sub-analysis of studies stratified by their risk of selection bias indicates that this bias may amplify the protective effect of influenza vaccination. The converse bias that people who are frailer are less likely to be vaccinated and more likely to suffer stroke (referred to as "frailty bias") is also potentially important [30], but few studies have adjusted for relevant confounding factors (e.g., general practitioner consultation rate).

Although self-reported vaccination history is reported to be highly accurate [31], our analysis indicates that problems in recall could inflate the observed protective effect of vaccination, as stroke patients might not accurately remember their vaccination status. Also relying on one's memory of the date of their influenza vaccination could result in immortal time bias. Therefore, agreement between self-reports and computerized databases is needed to confirm vaccination status in future studies. Furthermore, interview-based studies are also prone to selection bias because severe stroke patients (i.e., with aphasia or reduced consciousness) are ineligible.

Several mechanisms could explain the association between influenza vaccination and stroke risk. Compelling evidence suggests that nearly all types of infectious agents increase the incidence of stroke, and the risk of stroke might be associated with an aggregate viral burden rather than an individual pathogen, not excepting influenza [32]. The influenza-induced systemic inflammatory response precipitates vulnerable plaque rupture via altered concentrations of reactant proteins and cytokines, accelerates endothelial injury by impairing vasodilatation via metabolic derangements, and enhances thrombotic tendencies through altered clotting factors and platelet dysfunction [33]. In a study with apoE knockout mice, influenza vaccination resulted in atherosclerotic regions with lower lipid content and higher concentrations of smooth muscle cells and collagen [9].

The immune response to vaccination itself may be another mechanism by which vaccination confers a reduced risk of CVD. Vaccine-induced antibodies activate the bradykinin-2 receptor that induces nitric oxide production and vasodilatation, which may eventually result in atherosclerotic plaque stabilization [34]. However, this observation was made in animals with intact immune responses. Moreover, clinical studies exploring the effect of other vaccines could not support this hypothesis. In particular, a large case-series study showed that tetanus vaccination did not produce a detectable change in the risk of major CVDs [8], and Grau et al. [11] reported that other combined vaccinations were not associated with reduced risk of stroke.

Overall, ischemic strokes make up $80-90 \%$ of all stroke cases. Most pathogenic mechanisms of infection are more closely linked to ischemic strokes than hemorrhagic strokes, as inflammatory cascades promote atherosclerosis, plaque rupture, and thrombosis, leading to ischemic stroke [3]. Influenza vaccination may also protect against ischemic strokes such as cardiac embolism by reducing heart disease, which may prevent thromboembolic complications in the brain. By contrast, few published studies have assessed the association between infection and hemorrhagic strokes, although infection can contribute to aneurysmal rupture through endothelial instability via procoagulant mechanisms, fever, dehydration, and proteolytic lesions to the vessel wall [3]. One case-control study [35] identified a borderline association between subarachnoid hemorrhage and recent respiratory infection, and a larger prospective study [36] failed to detect an association with intracranial hemorrhage. To our knowledge, preventive effect of influenza vaccination against hemorrhagic stroke has been explored only in 2 studies, but neither reached significance $[11,20]$.

Given the seriousness of CVD morbidity, the effect size for influenza vaccination would be lower for a first stroke than for other stages of prevention. Well-designed studies show that vaccines are less effective in healthy people [37]. A recent meta-analysis also shows that the largest effect of influenza vaccination is seen among patients with the highest CVD risk [10]. A major caveat is that this finding was the result of "indication bias," in that individuals with higher CVD risk are more likely to be offered vaccination. However, our finding suggests that influenza vaccination could confer additional health benefits in terms of stroke prevention even in low-risk populations. 
This meta-analysis has several limitations. First, most studies were conducted in Western societies, whereas Asian and Western cultures differ notably with regard to stroke incidence and stroke-prone behaviors [38]. The pattern of vaccination uptake may also vary across countries, whereas studies included in our analysis were performed only in high-income countries with established influenza vaccination recommendations. Second, there were large variations across years in influenza activity, pathogenicity of circulating strains, and degree of vaccine-virus antigenic match. The effectiveness of annual influenza vaccines on CVD risk may vary depending on the vaccine match to circulating strains, although incompletely matched influenza vaccines still provide protection [39]. The timing of vaccination is also important, as the protective effect of influenza vaccination against stroke wanes over time following vaccination [40].

This meta-analysis of observational studies shows that influenza vaccination is associated with a lower risk of stroke events. Influenza vaccination is a readily available, inexpensive, and safe intervention that may reduce the risk of stroke. Our results underline current recommendations for annual influenza vaccination with the additional benefit of stroke prevention. Future randomized controlled trials are needed to confirm the protective effect of influenza vaccination on the risk of stroke.

\section{Disclosure Statement}

None declared.

\section{Funding Sources}

This study was supported by the Senior-friendly Product R\&D program funded by the Ministry of Health and Welfare thorough the Korea Health Industry Development Institute (HI14C1435).

\section{References}

1 Lloyd-Jones D, Adams R, Carnethon M, De Simone G, Ferguson TB, Flegal K, Ford E, Furie K, Go A, Greenlund K, Haase N, Hailpern S, Ho M, Howard V, Kissela B, Kittner S, Lackland D, Lisabeth L, Marelli A, McDermott M, Meigs J, Mozaffarian D, Nichol G, O'Donnell C, Roger V, Rosamond W, Sacco R, Sorlie P, Stafford R, Steinberger J, Thom T, Wasserthiel-Smoller S, Wong N, Wylie-Rosett J, Hong Y: Heart disease and stroke statistics-2009 update: a report from the American Heart Association Statistics Committee and Stroke Statistics Subcommittee. Circulation 2009;119:e21-e181.

2 Kunst AE, Amiri M, Janssen F: The decline in stroke mortality: exploration of future trends in 7 Western European countries. Stroke 2011;42:2126-2130.

3 Fugate JE, Lyons JL, Thakur KT, Smith BR, Hedley-Whyte ET, Mateen FJ: Infectious causes of stroke. Lancet Infect Dis 2014;14: 869-880.

4 Liu C, Yavar Z, Sun Q: Cardiovascular response to thermoregulatory challenges. Am J Physiol Heart Circ Physiol 2015;309:H1793H1812.

5 Palm F, Dos Santos M, Urbanek C, Greulich M, Zimmer K, Safer A, Grau AJ, Becher H: Stroke seasonality associations with subtype, etiology and laboratory results in the Ludwigshafen Stroke Study (LuSSt). Eur J Epidemiol 2013;28:373-381.

6 Barnes M, Heywood AE, Mahimbo A, Rahman B, Newall AT, Macintyre CR: Acute myocardial infarction and influenza: a metaanalysis of case-control studies. Heart 2015; 101:1738-1747.
7 Toschke AM, Heuschmann PU, Wood O, Wolfe CD: Temporal relationship between influenza infections and subsequent first-ever stroke incidence. Age Ageing 2009;38:100103.

8 Smeeth L, Thomas SL, Hall AJ, Hubbard R, Farrington P, Vallance P: Risk of myocardial infarction and stroke after acute infection or vaccination. N Engl J Med 2004;351:26112618.

9 Bermudez-Fajardo A, Oviedo-Orta E: Influenza vaccination promotes stable atherosclerotic plaques in apoE knockout mice. Atherosclerosis 2011;217:97-105.

10 Udell JA, Zawi R, Bhatt DL, Keshtkar-Jahromi M, Gaughran F, Phrommintikul A, Ciszewski A, Vakili H, Hoffman EB, Farkouh ME, Cannon CP: Association between influenza vaccination and cardiovascular outcomes in high-risk patients: a meta-analysis. JAMA 2013;310:1711-1720.

11 Grau AJ, Fischer B, Barth C, Ling P, Lichy C, Buggle F: Influenza vaccination is associated with a reduced risk of stroke. Stroke 2005;36: 1501-1506.

12 Nichol KL, Nordin J, Mullooly J, Lask R, Fillbrandt K, Iwane M: Influenza vaccination and reduction in hospitalizations for cardiac disease and stroke among the elderly. N Engl J Med 2003;348:1322-1332.

13 Lavallee P, Perchaud V, Gautier-Bertrand M, Grabli D, Amarenco P: Association between influenza vaccination and reduced risk of brain infarction. Stroke 2002;33:513518.

14 Hung IF, Leung AY, Chu DW, Leung D, Cheung T, Chan CK, Lam CL, Liu SH, Chu
CM, Ho PL, Chan S, Lam TH, Liang R, Yuen KY: Prevention of acute myocardial infarction and stroke among elderly persons by dual pneumococcal and influenza vaccination: a prospective cohort study. Clin Infect Dis 2010;51:1007-1016.

15 Pinol-Ripoll G, de la Puerta I, Santos S, Purroy $\mathrm{F}$, Mostacero E: Chronic bronchitis and acute infections as new risk factors for ischemic stroke and the lack of protection offered by the influenza vaccination. Cerebrovasc Dis 2008;26:339-347.

16 Newall AT, Kelly H, Harsley S, Scuffham PA: Cost effectiveness of influenza vaccination in older adults: a critical review of economic evaluations for the 50- to 64-year age group. Pharmacoeconomics 2009;27:439450.

17 Ajani UA, Ford ES, Okoro CA, Strine TW, Giles WH, Mokdad AH: Low prevalence of influenza vaccination among people with cardiovascular disease-BRFSS. Am J Prev Med 2005;29:31-35.

18 Stroup DF, Berlin JA, Morton SC, Olkin I, Williamson GD, Rennie D, Moher D, Becker BJ, Sipe TA, Thacker SB: Meta-analysis of observational studies in epidemiology: a proposal for reporting. Meta-analysis Of Observational Studies in Epidemiology (MOOSE) group. JAMA 2000;283:20082012.

19 Nicholas JM, Grieve AP, Gulliford MC: Within-person study designs had lower precision and greater susceptibility to bias because of trends in exposure than cohort and nested case-control designs. J Clin Epidemiol 2012; 65:384-393. 
20 Lin HC, Chiu HF, Ho SC, Yang CY: Association of influenza vaccination and reduced risk of stroke hospitalization among the elderly: a population-based case-control study. Int J Environ Res Public Health 2014;11:3639-3649.

21 Katz KA: The (relative) risks of using odds ratios. Arch Dermatol 2006;142:761-764.

22 Vamos EP, Pape UJ, Curcin V, Harris MJ, Valabhji J, Majeed A, Millett C: Effectiveness of the influenza vaccine in preventing admission to hospital and death in people with type 2 diabetes. CMAJ 2016;188:E342-E351.

23 Siriwardena AN, Asghar Z, Coupland CC: Influenza and pneumococcal vaccination and risk of stroke or transient ischaemic attackmatched case control study. Vaccine 2014;32: 1354-1361.

24 Lavallee PC, Labreuche J, Fox KM, Lavados P, Mattle H, Steg PG, Amarenco P: Influenza vaccination and cardiovascular risk in patients with recent TIA and stroke. Neurology 2014;82:1905-1913.

25 Puig-Barbera J, Diez-Domingo J, Varea AB, Chavarri GS, Rodrigo JA, Hoyos SP, Vidal DG: Effectiveness of MF59-adjuvanted subunit influenza vaccine in preventing hospitalisations for cardiovascular disease, cerebrovascular disease and pneumonia in the elderly. Vaccine 2007;25:7313-7321.

26 Grohskopf LA, Sokolow LZ, Broder KR, Olsen SJ, Karron RA, Jernigan DB, Bresee JS: Prevention and control of seasonal influenza with vaccines. MMWR Recomm Rep 2016;65:1-54.
27 Phrommintikul A, Kuanprasert S, Wongcharoen W, Kanjanavanit R, Chaiwarith R, Sukonthasarn A: Influenza vaccination reduces cardiovascular events in patients with acute coronary syndrome. Eur Heart J 2011;32: 1730-1735.

28 Jackson ML, Yu O, Nelson JC, Naleway A, Belongia EA, Baxter R, Narwaney K, Jacobsen SJ, Shay DK, Jackson LA: Further evidence for bias in observational studies of influenza vaccine effectiveness: the 2009 influenza A(H1N1) pandemic. Am J Epidemiol 2013; 178:1327-1336.

29 Jackson LA, Jackson ML, Nelson JC, Neuzil KM, Weiss NS: Evidence of bias in estimates of influenza vaccine effectiveness in seniors. Int J Epidemiol 2006;35:337-344.

30 Jackson LA, Nelson JC, Benson P, Neuzil KM, Reid RJ, Psaty BM, Heckbert SR, Larson EB, Weiss NS: Functional status is a confounder of the association of influenza vaccine and risk of all cause mortality in seniors. Int J Epidemiol 2006;35:345-352.

31 Mangtani P, Shah A, Roberts JA: Validation of influenza and pneumococcal vaccine status in adults based on self-report. Epidemiol Infect 2007;135:139-143.

32 Grau AJ, Urbanek C, Palm F: Common infections and the risk of stroke. Nat Rev Neurol 2010;6:681-694.

33 Naghavi M, Wyde P, Litovsky S, Madjid M, Akhtar A, Naguib S, Siadaty MS, Sanati S, Casscells W: Influenza infection exerts prominent inflammatory and thrombotic effects on the atherosclerotic plaques of apolipoprotein E-deficient mice. Circulation 2003;107:762768.
34 Veljkovic V, Glisic S, Veljkovic N, Bojic T, Dietrich U, Perovic VR, Colombatti A: Influenza vaccine as prevention for cardiovascular diseases: possible molecular mechanism. Vaccine 2014;32:6569-6575.

35 Kunze AK, Annecke A, Wigger F, Lichy C, Buggle F, Schnippering H, Schnitzler P, Grau AJ: Recent infection as a risk factor for intracerebral and subarachnoid hemorrhages. Cerebrovasc Dis 2000;10:352-358.

36 Roquer J, Cuadrado-Godia E, Giralt-Steinthauer E, Jimena S, Jimenez-Conde J, Martinez-Rodriguez JE, Ois A, Rodriguez-Campello A: Previous infection and stroke: a prospective study. Cerebrovasc Dis 2012;33: 310-315.

37 Simonsen L, Taylor RJ, Viboud C, Miller MA, Jackson LA: Mortality benefits of influenza vaccination in elderly people: an ongoing controversy. Lancet Infect Dis 2007;7:658666.

38 Kim AS, Johnston SC: Global variation in the relative burden of stroke and ischemic heart disease. Circulation 2011;124:314-323.

39 Dean AS, Moffatt CR, Rosewell A, Dwyer DE, Lindley RI, Booy R, MacIntyre CR: Incompletely matched influenza vaccine still provides protection in frail elderly. Vaccine 2010; 28:864-867.

40 Asghar Z, Coupland C, Siriwardena N: Influenza vaccination and risk of stroke: self-controlled case-series study. Vaccine 2015;33: 5458-5463. 